\title{
Die Entwicklung der sozio-politischen Artikulationsfähigkeit der Ureinwohner Australiens
}

\section{Einleitung}

Ein immer wieder diskutierter Problembereich auf dem australischen Kontinent betrifft die Beziehungen der indigenen Bevölkerung zu anderen Bevölkerungsgruppen, ihre Rechte und ihre Stellung innerhalb der Gesellschaft. Die Kontroverse von nationaler Einheitlichkeit und den Rechten bzw. Privilegien von Minderheiten gehört zu den dringendsten Angelegenheiten, die auf der australischen politischen Ebene zur Zeit diskutiert werden. Aktuelle Bestrebungen, ein Abkommen betreffend die Aborigines zu entwerfen, stoßen auf Schwierigkeiten, welche tief in der Geschichte des Kontinentes und im Versuch einer Abgrenzung der Identität der Ureinwohner verwurzelt sind. Die unter den Mitgliedern der regierenden Labour Party (Arbeiterpartei) der verschiedenen Länder des australischen Bundes bestehenden Uneinigkeiten über den Inhalt eines solchen «Treaty» stellen eine weitere Hürde dar.

Mitte der 80er Jahre geriet die mächtige Bewegung für Landrechte der Ureinwohner ins Wanken, da die Landesregierungen von Western Australia, Queensland und des Northern Territory beschlossen, der Last der von den Hauptstädten des südöstlichen Landesteiles gegen Mißhandlung der Aborigines versprochenen Kompensationsdialektik der 70er Jahre zu widerstehen. Bei den größeren peripheren Bundesstaaten bestand kein Interesse, das Gewissen der entfernten Großstädte auf eigene Kosten zu beruhigen - obwohl durchwegs anerkannt ist, da $B$ die Ureinwohner Australiens Opfer grober Menschenrechtsverletzungen waren und sind. Um einen Ausgangspunkt für unsere Diskussion zu schaffen, wird an dieser Stelle ein kurzer Überblick der wichtigsten rechtlichen und politischen Ereignisse der letzten Jahre, welche auf die Entwicklung der zeitgenössischen Aboriginal-«Nation» wirkten, gegeben.

\section{Die indigene Bevölkerung Australiens}

Die Aborigines sind das Volk, welches sich in Australien - mindestens 42000 Jahre vor dem Eintreffen der Weißen - an das Land anpaßte und dieses in der Folge bewohnte. Ein Kernpunkt der hiesigen Diskussion betrifft die Frage: Wer ist heute eigentlich ein Aborigine? Es kann kein Abkommen geschlossen werden, ohne definieren zu können, ob und inwiefern ein Bruchteil von «Aboriginal»-Abstammung genügt, um den Betroffenen Sonderrechte und Kompensationen zu gewähren. In der Rede eines Vertreters der australischen Regierung über «die Rechte der indigenen Völker», am 30. Juli 1984 vor der UNO-Arbeitsgruppe für indigene Völker in Genf gehalten, wurde das Formulieren von Kriterien, mittels derer sich die betreffenden Völker zu identifizieren vermöchten, grundsätzlich den Ureinwohnern selbst überlassen. Dennoch existiert eine provisorische Definition, welche zur Ausführung von Regierungsprogrammen dient und die drei Schlüsselelemente beinhaltet. Eine Person muß:

- von der Herkunft «aboriginal» sein

- sich selbst als Aborigine identifizieren

- von der Aboriginal-Gemeinschaft, mit der sie verkehrt, als Aborigine akzeptiert sein.

Durch die Einführung dieser Abgrenzungselemente erhoffte sich die australische Regierung die Vermeidung von «einschränkenden, potentiell beleidigenden Kriterien wie Herkunftsgrad»!

\section{Die sozial-politische Stellung der Aborigines}

Aborigines sind seit 1788 weißem Rassismus, Mißhandlungen sowie politischen und rechtlichen $\mathrm{Be}$ nachteiligungen ausgesetzt. Ihre rechtliche Lage und ihr Bedürfnis nach Fürsorge sind erst in den 60er Jahren dieses Jahrhunderts zu einem politischen Diskussionspunkt geworden. Erst seit dem Volksentscheid («people's vote») von 1967, bei dem die entsprechende Vorlage von 91 Prozent der Wählerschaft und von allen sechs Ländern des Bundes angenommen wurde, sind die Aborigines in vollem Umfange als australische Staatsbürger anerkannt. Der australische Bund erlangte die Kompetenz, Gesetze betreffend die Aborigines zu erlassen, und

Cheri Ragaz, Dr., Geographisches Institut, Universität Zürich-Irchel, Winterthurerstraße 190, 8057 Zürich 
wurde verpflichtet, die Aborigines bei Volksabstimmungen künftig mit einzubeziehen. Von den neuen Kompetenzen wurde aber bis 1972 , als die Regierung unter $\mathrm{G}$. Whitlam die erste staatliche Abteilung für «Aboriginal»-Angelegenheiten gründete, kaum Gebrauch gemacht. Während der 70er Jahre begannen kleine Gruppen von Ureinwohnern und Fürsorgeorganisationen Druck auf die australische Regierung auszuüben. Sie äußerten sich besonders lautstark über ihre Rechtsansprüche auf das Land. Diese Zeit war auch durch eine zunehmende Internationalisierung des Kampfes der Aborigines gekennzeichnet. In den späten 70er Jahren reisten beispielsweise mehrere Abgeordnete der Aborigines nach Europa. 1981 untersuchte der Weltkirchenrat die Lage der Aborigines in Australien und warf der Regierung und dem Volk Rassismus und Mißhandlung der Ureinwohner vor.

Die 1972 geschaffene Abteilung für Angelegenheiten der Aborigines sollte die Kontrolle durch die bisher zuständigen Landesabteilungen durch eine solche auf nationaler Ebene ersetzen und für politische Maßnahmen und Geldzuwendungen zugunsten der Aborigines zuständig sein. Diese Neuerung wurde, außer im Bundesstaat Queensland, auch durchgeführt. Direkte Konfrontationen zwischen den Landesteilen und dem Bund konnten damit vermieden werden. Die Geschäfte der Abteilung für Angelegenheiten der Aborigines wurden 1987 der A.D.C. (Australian Development Commission) überwiesen.

\section{Demographische Übersicht}

Die Volkszählung von 1981 ergab eine Zahl von etwa 160000 Aborigines, die damit etwa 1 Prozent der Gesamtbevölkerung stellten. In bezug auf die einzelnen Bundesstaaten ergab sich folgende Verteilung: 29 Prozent der Aborigines lebten in Queensland, 22 Prozent in New South Wales, 20 Prozent in Western Australia, 18 Prozent im Northern Territory, 6 Prozent in South Australia und 4 Prozent in Victoria. Etwas weniger als 1 Prozent hielten sich in A.C.T. und Tasmania auf. 58 Prozent der Aborigines waren in den urbanen Räumen wohnhaft. Das mittlere Familieneinkommen betrug 6000 australische Dollar - etwa die Hälfte desjenigen einer weißen Familie. Die Kindersterblichkeitsziffer war mit 26 Prozent mehr als doppelt so hoch wie die der weißen Einwohner. Auch die durchschnittliche Lebenserwartung der Aborigines lag mit 55 Jahren um 20 Jahre tiefer als bei der weiBen Bevölkerung. Die Arbeitslosigkeitsziffer der Aborigines von 25 Prozent ergab eine viermal höhere Zahl als bei den Weißen, und die sogenannte «Gefängnisziffer» (775 pro 100000$)$ war in bezug auf die Aborigines sogar elfmal höher.

\section{Ansprüche auf das Land}

Wie schon angedeutet, ist als Kernpunkt der Identität der Aborigines deren implizierte Untrennbarkeit mit dem Land zu betrachten. Der Begriff «Landrechte» bezieht sich auf aboriginale Forderungen nach Wiedergewinnung oder Ersatz der traditionellen heiligen Gebiete, welche von den WeiBen übernommen worden waren. 1963 übergaben die Yirrkala-Stämme der Bundesregierung eine auf Baumrinde geschriebene Bittschrift (petition) für ihr Land. Im Jahre 1972 wurde auf dem Gelände des Regierungssitzes in Canberra eine "Zeltbotschaft» aufgeschlagen, welche breites öffentliches Aufsehen erregte. Die Ansprüche der Aborigines auf das Land wurden erst 1973/74 unter «weißem Recht» anerkannt, als die «Aboriginal Land Rights Commission» unter Richter A. C. Woodward die Übertragung von Land im Northern Territory auf die ursprünglichen Besitzer empfahl. 1976 folgte die entsprechende Gesetzgebung, auf Grund welcher die Wattie Creek-Region des Northern Territory an die Gurindji-Leute zurückgegeben wurde.

Die genannte Kommission gründete die "Aboriginal Land Commission», welche u. a. zur Aufgabe hatte, Besitzansprüche zu überprüfen und im Norden sowie im Zentrum «Land Councils» einzurichten. Die Aboriginal Land Rights Act von 1976 wurde von vielen Kreisen als bahnbrechend angesehen, obwohl gewisse Aboriginal-Gruppen die Meinung vertraten, daß es sich dabei nur um die Rückgabe traditioneller Ländereien handelte, welche Teile von nicht veräußerten (unalienated) Krongütern oder übertragenes, schon von den Aborigines gekauftes Land darstellten. Zur Zeit besitzen die Aborigines etwa 30 Prozent der Landfläche des Northern Territory. Es handelt sich dabei zum größten Teil um dürres, unproduktives Land. Forderungen der Aborigines nach fruchtbarem Land sind bisher von der Bergbauindustrie, der Viehwirtschaft und der Regierung des Northern Territory abgewiesen worden.

Im Jahre 1987 übertrug die Regierung von South Australia große und bedeutsame Landflächen sowie die Kontrolle über den Bergbau den Pitjantjatjara Aborigines. New South Wales genehmigte 1984 Landrechtsgesetze, brachte aber 1986 einen Zusatzantrag ein, wonach Umfang und Sicherheit des Besitzanspruchs der Aborigines streng begrenzt wurden. Die Regierung von Queensland trat im Kampf für die Gewährung von Landrechten an die Aborigines stets als Gegnerin auf, versprach 1987 jedoch eine Erweiterung der Gesetzgebung zugunsten von Landrechten für die Ureinwohner. Die in Victoria beantragte Gesetzgebung wurde wegen Opposition sowohl von weißer Seite wie auch von den Aborigines bereits zweimal umgeschrieben. Die Regierung von Western Australia zog sich von ihren früher ge- 
machten Versprechungen zurück, bekräftigte dafür, zusammen mit der Bundesregierung die Dienstleistungsorganisationen der Aborigines finanziell zu unterstützen. Tasmania besitzt keine Gesetzgebung über Landrechte für Ureinwohner. Verschiedene Regierungen des australischen Staatenbundes haben sich bisher zu diesen Rechtsfragen nur sehr zurückhaltend geäußert. Die 1985 zu beobachtenden Bestrebungen der Staatsregierung zur Schaffung von gesetzlichen Grundlagen für die Anerkennung einheitlicher Landrechte begegneten seitens der Landesregierungen, insbesondere von Western Australia, starkem Widerstand. Das Thema «Landrechte» bleibt jedenfalls ein strittiger und oft mißverstandener Diskussionspunkt.

\section{Die Konsequenzen der Landübertragung}

Die Bewegung für Landrechte hat den Ureinwohnern Australiens mehr Autonomie und Selbstbestimmung verschafft als irgendwelche andere politische Maßnahmen. Die schon erwähnte Aboriginal Land Rights (Northern Territory) Act von 1976 ist bedeutungsvoll - aber auch umstritten. Die Akte sprach nicht nur den Aborigines große Landflächen zu, sondern ermöglichte durch die Gründung von gesetzgebenden Körperschaften, die ausreichend finanziert und damit unabhängig wurden, auch ihr freies Handeln. Es wurde anerkannt, $\mathrm{da} B$ es sich beim Streitpunkt «Landrechte» nicht nur um Besitzansprüche, sondern auch um die Wiederherstellung eines Teils der persönlichen und der Gruppenautonomie handelte, welche in vorkolonialen Zeiten vorhanden gewesen war. Über rechtliche und administrative Fragen in bezug auf die Aborigines ist in Australien stets viel geschrieben worden. Andererseits war schon immer ein großer Unwille vorhanden, die Auswirkungen der Kolonialherrschaft und die Art des Landbesitzes in vorkolonialer Zeit, worauf die Aborigines die Kontinuität ihrer Beziehung zu ihrem Geburtsland (country) stützen konnten, zu anerkennen.

\section{Herkömmliche Rechtssysteme der Aborigines im Vergleich}

Um einen Überblick über den Landbesitz der Aborigines zu gewähren, erscheint es zweckmäßig, folgende Ausgangspunkte darzulegen: Erstens ist das Wissen um die traditionellen Prinzipien des Landbesitzanspruchs erforderlich, um die Identitätsentfaltung der Aborigines verstehen zu können. Zweitens müssen die Konsequenzen von Interaktionen zwischen Eingeborenen und Weißen in bezug auf Landbesitzsysteme aufgezeigt werden. Diese Phase ist durch intensive Auseinandersetzungen zwischen Aborigines und Experten der Rechtswissenschaft, der Anthropologie und der Soziologie gekennzeichnet. An dieser Stelle muß betont werden, daß das "Australian Aboriginal Customary Law» vom europäischen Rechtssystem nicht anerkannt worden ist. Für die Ureinwohner stellt sich das Problem, sich mit den europäischen Rechtsbegriffen auseinandersetzen zu müssen. Das traditionelle Recht der Aborigines ist stark von Verwandtschaftssystemen abhängig und variiert von Region zu Region. Es beinhaltet keine formalen Gesetze; in vielen Fällen sind kollektive Meinungen maßgebend. Der jeweils geschlossene kulturelle Rahmen, innerhalb dessen diese Rechtsbegriffe damals entstanden, ist heute meist zerbrochen. 1977 wurde der "Australian Law Reform Commission" die Aufgabe übertragen, die potentiell rechtsgültigen (im europäischen Sinne) Aspekte der Gemeinschaft der Aborigines zu identifizieren. Der 1986 publizierte Bericht empfahl eine sehr begrenzte Anerkennung des «Aboriginal Australian Customary Law»; die Bundesregierung veröffentlichte bisher keine Stellungnahme dazu.

Drittens betrifft ein weiterer Ausgangspunkt die Schaffung neuer administrativer Einheiten, wie etwa Landräte innerhalb des landrechtlichen Kontexts. Schließlich stellt sich angesichts von Bergbau und anderen Aktivitäten die Frage nach der Verwaltung von zurückerhaltenem Land durch die Aborigines. Diese Frage stellt in der Geschichte der Ureinwohner ein besonders wichtiges Kapitel dar.

\section{Traditionelle Konzepte der Aborigines}

Die traditionelle Kosmologie der Aborigines nimmt an, daß Natur und Kultur gleichzeitig geformt worden sind - durch Mächte oder Kräfte, welche die Welt während der Traumzeit (oder Urzeit) besiedelt haben und immer noch besiedeln, obwohl sie nicht mehr sichtbar sind. Diese Theorien entstehen aus Raum- und Zeit-Überlegungen, und nicht durch Schöpfung aus dem Nichts. Maddock schreibt: "Aboriginal cosmology is more than a theory of the origin and structure of the world: it includes a theory of human action»1. Es besteht die Auffassung, da $\beta$ die Erde und das Leben schon existent waren, als die Mächte ihre Tätigkeiten aufgenommen haben. Sie formten die Landschaft, in der die Menschen verweilen, sie gestalteten die Spezien, mit denen sie leben und wovon sie leben, und (die Mächte) errichteten einen Plan des Lebens, worin die Menschen sich fügen. Die Kommunikations-

\footnotetext{
1 Maddock, Kenneth; The Australian Aborigines. A Portrait of their Society. Melbourne 1972. S. 132.
} 
wege, welche auf beiden Seiten dieser Dualität bestehen, sind komplex und nur unter außerordentlichen Bedingungen offen.

Maddock suggeriert, daß die Aborigines das Gefüge der Welt nach den sozialen Strukturen formten, deren Plan schon in der Traumzeit schöpferisch festgelegt worden war. Der Mensch reproduziere bloß Formen und stelle gemäß einem Plan Ordnung her. Die Aborigines glauben, da $ß$ die menschlichen Rechte auf das Land genau gleich alt wie die heutige Gestalt der Erde seien, und daß sie aus den Persönlichkeiten entstanden, welche der Welt ihre Form gegeben hätten. Wenn etwas zum Besitz erklärt werden könnte, dann wäre es der heilige religiöse Diskurs, der auf eine bestimmte Lokalität Bezug nahm und einem Mitglied eines bestimmten "Patriclans» bekannt war. Gruppen, die mit diesen Lokalitäten verbunden sind, haben eine korporative Identität, indem sie, trotz Verlust ihrer Mitglieder durch den Tod, über die Zeit hinweg fortbestehen können.

\section{Theoretische Überlegungen}

Im Laufe der Zeit reagieren menschliche Gesellschaften auf innere und äußere Stimuli. Diese Reaktionen hängen von der Wahrnehmung und von den Entscheidungen der Gemeinschaft $a b$, gleich ob die Stimuli real sind oder nicht. Eine solche Entwicklung stellt über die Zeit hinweg eine Kulturanpassung dar. Zwischen generellen Umwelteinflüssen und adaptiven Mustern kann keine Kausalität bestehen. Adaptive Folgerungen auf eine exogene Veränderung können nicht vorausgesagt werden. Ein offener sozio-kybernetischer Rahmen würde die folgenden Komponenten einbeziehen:

1) Umwelt (der Begriff «Umweltvariablen» beinhaltet die Voraussetzung, daß irgendwelche Aspekte des menschlichen Handelns direkt in eine Wechselwirkung mit der Umwelt treten können).

2) Erkenntnisvermögen (die Wahrnehmung und Verarbeitung von Informationen).

3) Verhalten oder Handeln (Muster der menschlichen Interaktion in Raum und Zeit).

4) Gruppenidentität (Individuen als bewußt funktionierende Teile von Gruppen und deren Epistemologien).

Die Ureinwohner sind im Begriff, eine Ideologie zu entwickeln und zu bestätigen, welche einem reichen Erbe huldigt, die aber auch deutlich artikuliert worden ist und heute in Australien erlebt werden kann. Viele traditionelle Aspekte der Kultur werden nicht mehr praktiziert, das heißt, sie haben im zeitgenössischen System als wichtiger Bestandteil zu funktionieren aufgehört. Dennoch kommt ihnen in der Formation einer Gruppenidentität eine Hauptrolle zu. Es wird damit ein Volk bezeichnet, dessen Entwick- lung auf einer sozio-kulturellen Grundlage stattfand, die ein stabiles internes Gerüst für das Werden von Aboriginalität gewährleistete. Innerhalb eines sich verändernden human-ökologischen Bereichs, der Sprach-, Religions- und Kunstsystemkomponenten, zusammen mit weiteren Ausdrucksformen des menschlichen Geistes, beinhaltet, entsteht ein Identitäts- und Zusammengehörigkeitsgefühl, welches auf einer langen Geschichte basiert und dessen Kontinuität abgesichert ist. Menschliches Handeln auf lokaler Ebene stützt sich auf Erfahrungen, die einem Ort Charakter und Bedeutung verleihen.

\section{Zwischengesellschaftliche Konflikte}

Selbst in einem urbanen Raum wie Sydney prägt das kulturelle Erbe heute noch mehr oder weniger verbindlich das Individuum und sein Handeln. Die gewalttätigen Zusammenstöße zwischen Aborigines und der Polizei am 27. August 1988 in Redfern, einem Vorort von Sydney, N.S.W., sind ein Anzeichen für ein tief liegendes «malaise» der Gesellschaft. Am Tage danach führte der Polizeiminister in einem Interview aus, ein Teil der Verantwortung liege bei den früheren Regierungen, die es versäumt hätten, die Hauptproblembereiche Erziehung, Gesundheit und die Wohnungsfrage für die Aborigines zu lösen. Weiter erklärte er: «Wir müssen jetzt Positives tun und zum Beispiel darauf bestehen, daß die Kinder der Aborigines auch tatsächlich zur Schule gehen anstatt wegzubleiben. Es muß betont werden, daß die Aborigines mehr Verantwortung für ihre eigene Wohlfahrt und ihr eigenes Handeln übernehmen sollten.»2

Gesamthaft gesehen fehlt es heute am Verständnis für die Beweggründe, welche das Handeln der Aborigines konstituieren oder beeinflussen, für die traditionellen Prinzipien, welche die Gesellschaft immer noch, in bewußtem oder auch unbewußtem Sinne, prägen.

\section{Schlußwort}

Der Mensch handelt als ein historisches Wesen. Die Stätten werden innerhalb des menschlichen Bewußtseins zum Ereignis, zu einem kulturellen Konzept, dessen Charakter immer werdend ist. Die Industrialisierung und die Urbanisierung haben eine zunehmende Mobilität, ein eigentliches Aufkommen von neuen kulturellen Verbindungen, hervorgerufen. Soziale Unternehmungen (action) seitens

2 The Sunday Telegraph, Sydney, 28. August 1988. S. 2. 

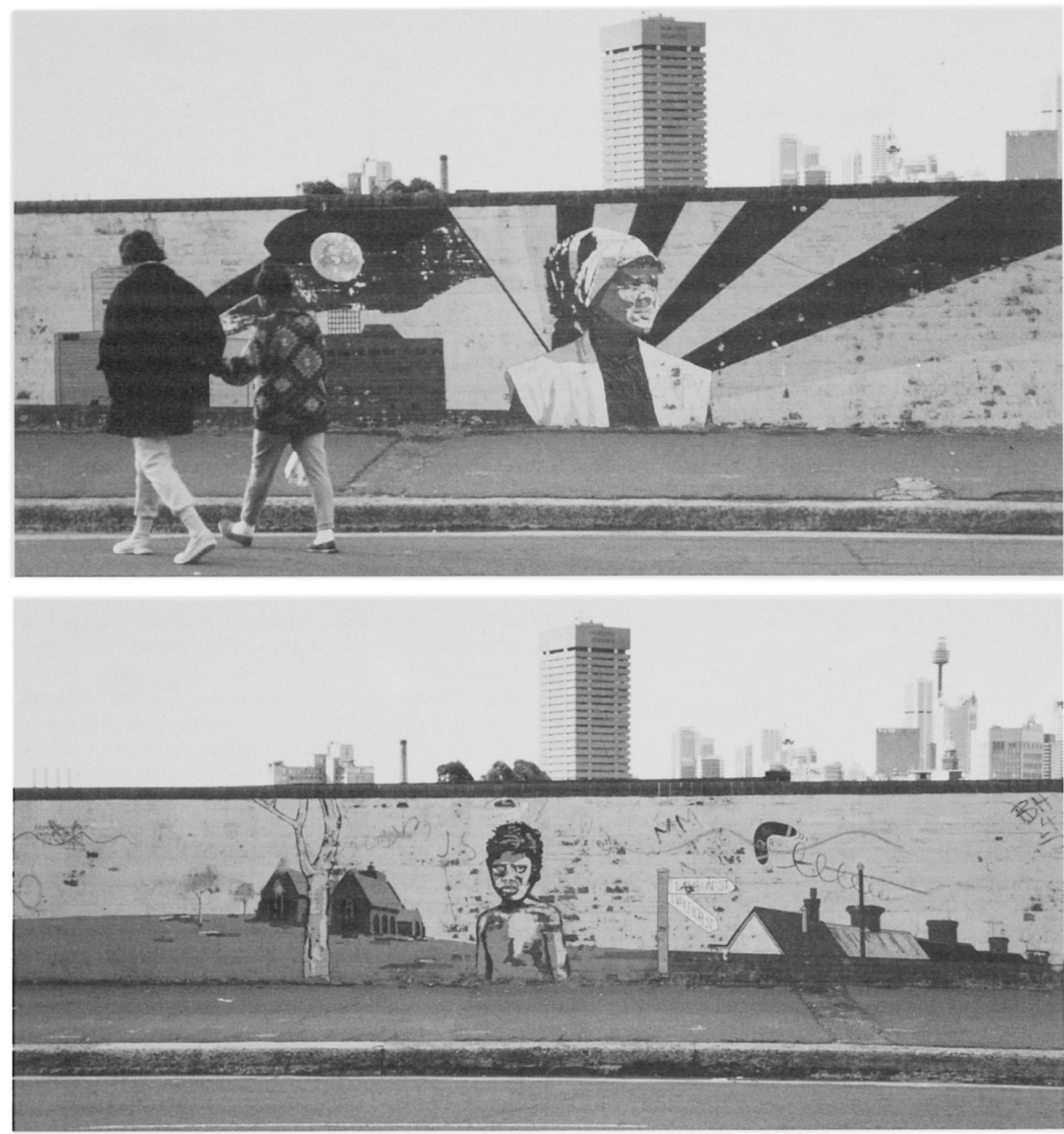

Abb. $1+2: \quad$ Wandmalereien als Ausdrucksmöglichkeit der urbanen Aborigines; hier zwei Beispiele aus Redfern, N.S.W., einem Vorort von Sydney, Aufnahme C. Ragaz, August 1988.

der Aborigines selbst verändern zur Zeit bestimmte Machtverhältnisse innerhalb der Gemeinschaft, welche aber auch soziale Erzeugnisse der Gesamtgesellschaft sind.

Die koloniale Elite, welche die australischen Ureinwohner dominierte (gemäß ihrem eigenen Begriff der «natürlichen» Gesetze des ökonomischen Wachstums und der neo-klassischen Historie), konnte den «ésprit» der Aborigines jedoch nicht zum Verschwinden bringen.

\section{Literatur:}

Land Rights Now - The Aboriginal fight for Land in Australia. In: IWGEA Document No. 54. Kopenhagen, 1985

MADDOCK, K. (1972): The Australian Aborigines. A portrait of their Society. Melbourne.

Speech to the United Nations Working Group on Indigenous Populations - The Rights of Indigenous People. Genf, 30.7. 1984

The Sunday Telegraph, Sydney, 28.8.1988 